\title{
Research on Distribution of Flow Field and Simulation of Working Pulsation Based on Rotating-Sleeve Distributing-Flow System
}

\author{
Yanjun Zhang, ${ }^{1}$ Hongxin Zhang, ${ }^{1,2}$ Jingzhou Yang, ${ }^{1}$ Qinghai Zhao, ${ }^{1,2}$ Xiaotian Jiang, \\ Qianchang Cheng, ${ }^{1}$ and Qingsong Hua ${ }^{1,2}$ \\ ${ }^{1}$ Mechanical and Electrical Engineering College, Qingdao University, Qingdao 266071, China \\ ${ }^{2}$ Power Integration and Energy Storage Systems Engineering Technology Center, Qingdao University, Qingdao 266071, China \\ Correspondence should be addressed to Hongxin Zhang; qduzhx@126.com and Qinghai Zhao; zqhbit@163.com
}

Received 21 May 2017; Revised 2 August 2017; Accepted 9 August 2017; Published 1 November 2017

Academic Editor: Agostino Bruzzone

Copyright (c) 2017 Yanjun Zhang et al. This is an open access article distributed under the Creative Commons Attribution License, which permits unrestricted use, distribution, and reproduction in any medium, provided the original work is properly cited.

To solve problems of leakage, vibration, and noise caused by disorders of flow field distribution and working pulsation in the rotating-sleeve distributing-flow system, governing equations of plunger and rotating sleeve and computational fluid dynamics (CFD) model are developed through sliding mesh and dynamic mesh technology to simulate flow field and working pulsation. Simulation results show that the following issues exist: obviously periodic fluctuation and sharp corner in flow pulsation, backward flow when fluid is transformed between discharge and suction, and serious turbulence and large loss in kinetic energy around the damping groove in transitional movements. Pressure in the pump chamber rapidly rises to $2.2 \mathrm{MPa}$ involving over $10 \%$ more than nominal pressure when the plunger is at the Top Dead Center (TDC) considering changes about damping groove's position and flow area in two transitional movements. Shortly pressure overshoot gradually decreases to a normal condition with increasing flow area. Similarly, pressure in the pump chamber instantaneously drops to a saturated vapor pressure $-98.9 \mathrm{KPa}$ when the plunger is at the Bottom Dead Center (BDC). With increasing flow area the overshoot gradually increases to the normal condition. This research provides foundations for investigating flow field characteristic and structure optimization of rotating-sleeve distributingflow system.

\section{Introduction}

A distributing-flow system, the most important component of hydraulic system, is widely used in the fluid power industry because of robustness, controllability, and wide operating range. However, the distributing-flow system controlled flow by valves has many disadvantages including bulk mass, large pressure loss, noise, and going against high frequency, which can easily cause noise and vibration on account of flow pulsation [1]. To solve these issues, the novel rotating-sleeve distributing-flow system is proposed which uses reciprocating motion of plunger and single-track rotation of rotating sleeve to achieve distribution functions. It has reliable seal, high efficiency, and little impact from working frequency.
To further improve its performance, the flow characteristics of rotating-sleeve distributing-flow system could be theoretically investigated; then the system efficiently reduces damage to the pump, such as undesirable noise, vibration, and cavitation even reducing working reliability of the pump [24].

Flow ripple, a significant characteristic of piston pump, is closely relevant with the pressure fluctuation, backward flow, and noise of inner fluid. Noises in pump can be effectively reduced by taking factors influencing the flow ripple into account and focusing on flow ripple in the process through structure optimization [5]. Dhananchezhiyan and Hiremath [6] reported that the flow ripple is associated with pressure pulsation in various drive frequencies of micro pumps. 
Hence, intensive studies of the flow ripple and the pressure pulsations are necessary for better understanding the flow process [7].

Recently, various analytic and simulative methods have been extensively studied on distribution of flow field and working pulsation. Particularly, the CFD simulation is generally utilized in many fluid field and hydraulic researches. Luo et al. [8] developed an adiabatic dehumidifier model using CFD technology and the interior heat and mass transfer processes were then simulated. Ma et al. [9] utilized a new CFD model with user defined function to simulate pump's fluid characteristic and predict the flow ripple. At the same time, the flow ripple was tested under different working parameters such as rotation speed and working pressure. Delele et al. [10] conducted research on CFD model based on an Eulerian-Eulerian multiphase which can predict the fluid flow profile and study the effects of drum rotational speed. In addition, they used experimental results of particle and fluid velocities and residence time to verify the model simulation.

The slide mesh and dynamics grid technology have been continually employed in CFD model to improve its simulation functions and apply varied motions into the model flexibly. Wang [11] utilized the dynamics CFD model to simulate cavitation of axial piston pump, which uses compressible fluid pump model with nine pistons. Guo et al. [12] studied the sliding mesh method of Fluent ${ }^{\circledR}$ to simulate the dynamic behavior in the course of ball valve closure. In addition, Vitagliano et al. [13] ran a slide mesh generated in zones of the flow field and connected with sliding boundaries under different rotation speed conditions to simulate unsteady flows with surfaces in relative motion.

Fortunately, some researchers have found effective ways to reduce working pulsation to decrease noise. Lee et al. $[14,15]$ investigated the computed time-accurate pressure field and the loss generation process to establish the causal link to the induced flow ripple in the turbine system. Alves et al. [16] found that combining CFD modeling and analytical techniques is a great way to predict the oil flow rate in the eccentric-tube centrifugal oil pumping system. The research group led by Palmberg set a precompression volume in valve plate between the discharged kidney slot and sucked kidney slot [17]. They found that small precompression volume can amazingly lessen enormous problems of noise, pulsation, and hydraulic impact $[18,19]$. And an axial piston pump's valve plate which adopts a prepressurization fluid path consisting of a damping hole, a buffer chamber, and an orifice can reduce flow ripple to some extent [20].

This paper aims to reduce vibration and noise by developing a novel rotating-sleeve distributing-flow system and analyzing the relationship between turbulence energy, velocity, and working pulsation through CFD simulation. In addition, the influences with respect to backward flow, flow pulsation, and pressure fluctuation are investigated. A complete simulation model with relevant parameters have been established and flow characteristics can be explicitly described. Furthermore, this work provides theoretical foundation for structure optimization of distributing-flow system and performance improvement.

\section{Operating Principle of Rotating-Sleeve Distributing-Flow System}

In this section, a novel rotating-sleeve distributing-flow system is developed and analyzed. The plunger pulled by the crank-link mechanism achieves coupled reciprocating movement and then uses drive pin to transmit force into rotating sleeve along the cam groove pathway in unidirectional rotating movement. The drive pin moves while rolling with the cam groove molded line obtained by fitting linear equation through quadratic differential, rotating angle of rotating sleeve, and crank angle. Figure 1 indicates the operating principle and components of rotating-sleeve distributingflow system for mass flow with high frequency and efficiency.

In this system, there are two major movements: axial reciprocating movement of the plunger and unidirectional rotating movement of rotating sleeve. The plunger finishes reciprocating movements powered by the crankshaft and connecting rod mechanism via a connector cross slider. The displacement of plunger in reciprocating motion is formulated as follows:

$$
x=r\left(1-\cos \varphi+\frac{\lambda}{2} \sin ^{2} \varphi\right),
$$

where $r$ is the radius of bent axle, $\varphi$ is crank angle about the crankshaft and connecting rod mechanism, $\lambda=r / l$ is the ratio of crank and connecting link, and $l$ is link length.

Due to $\varphi=\omega t$, the derivative of the displacement of plunger $x$ with respect to the time $t$ can be expressed as follows:

$$
u=r \cdot \omega \cdot\left(\sin \varphi+\frac{\lambda}{2} \sin 2 \varphi\right),
$$

where $\omega$ is angular velocity of bent axle.

Angular velocity and acceleration of rotating sleeve have no obvious phase step and inflection point when sine molded line is selected for cam groove. Therefore, the relationship between the cam groove's axial displacement and rotatingsleeve angle is given as follows:

$$
z(\theta)=\frac{S}{2}(1-\cos \theta) \quad(0 \leq \theta \leq \pi),
$$

where $S$ is plunger stroke and $\theta$ is the rotating-sleeve angle rotating around the central axis.

Substituting (1) into (3), the rotating-sleeve angle $\theta$ with respect to crank angle $\varphi$ is obtained as follows:

$$
\theta= \begin{cases}\arccos \left(\cos \varphi-\frac{\lambda}{2} \sin ^{2} \varphi\right), & 0 \leq \varphi \leq \pi \\ 2 \pi-\arccos \left(\cos \varphi-\frac{\lambda}{2} \sin ^{2} \varphi\right), & \pi \leq \varphi \leq 2 \pi .\end{cases}
$$

The angular velocity of rotating sleeve by taking the derivative of $t$ in (3) can be expressed as follows:

$$
\omega_{t}= \pm \frac{\omega}{\sqrt{1-C_{0}^{2}}} \cdot\left(\sin \varphi+\frac{\lambda}{2} \sin 2 \varphi\right)
$$

where $\omega_{t}$ is a positive number during $0 \leq \varphi<\pi$ and $\omega_{t}$ is a negative number during $\pi \leq \varphi<2 \pi$ and $C_{0}=\cos \varphi-$ $0.5 \lambda \sin ^{2} \varphi$. 


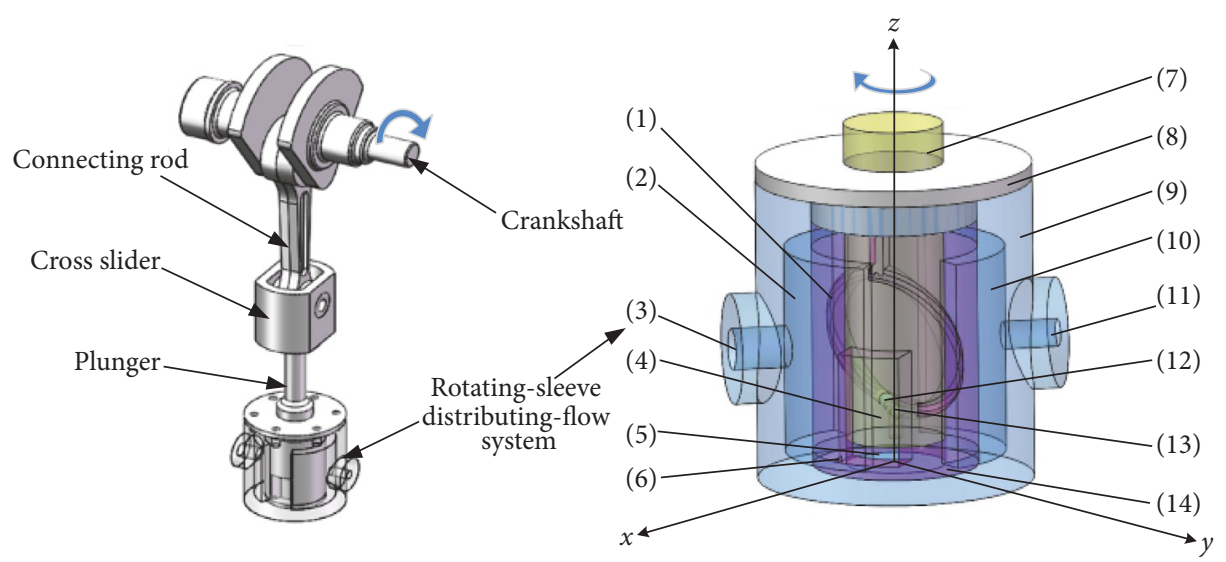

FIGURE 1: Structure principle of rotating-sleeve distributing-flow system. (1) Cam groove; (2) loading chamber; (3) inlet; (4) valve port; (5) pump chamber; (6) damping groove; (7) plunger; (8) blind flange; (9) pump body; (10) collecting chamber; (11) outlet; (12) drive pin; (13) compression spring; (14) rotating sleeve.

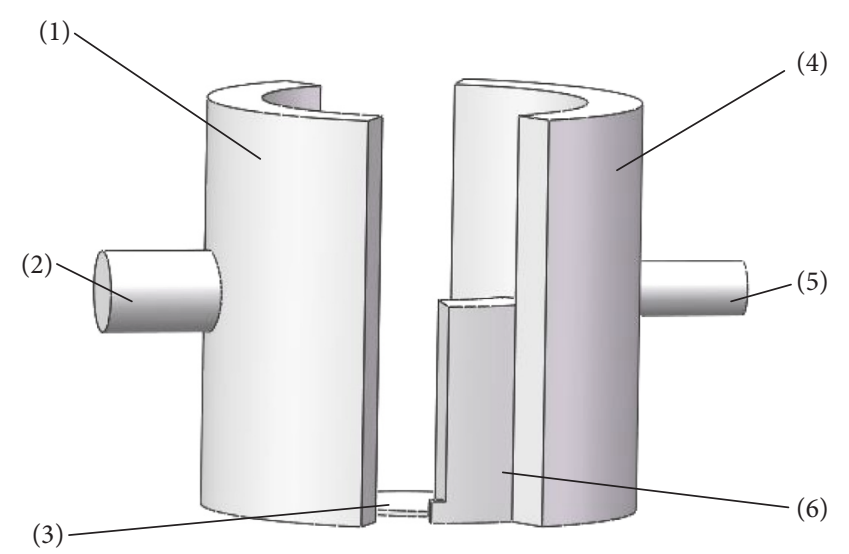

Figure 2: Fluid model of rotating-sleeve distributing-flow system. (1) Loading chamber; (2) inlet; (3) pump chamber; (4) collecting chamber; (5) outlet; (6) valve port.

\section{Models and Methods}

3.1. Fluid Model. In light of structure and operating principle of rotating-sleeve distributing-flow system, the fluid model is established as shown in Figure 2, in which the model indicates the main components with loading chamber, inlet, pump chamber, collecting chamber, outlet, and rotating sleeve.

In this paper, the fluid model is simulated and analyzed by fluid simulation software Fluent ${ }^{\circledR}$. The motions of plunger and rotating sleeve are defined in fluid model using Users Defined Function. Standard $k-\varepsilon$ turbulence model and SIMPLE arithmetic are applied into simulation settings. Technologies of sliding mesh and dynamic mesh are also utilized in the fluid model according to its specific motion characteristics. The model parameters and boundary conditions are defined in Table 1.

3.2. Cavitation Model. The cavitation model is based on the flow equation Navier-Stokes with variable density and standard viscosity in hydromechanics. In this paper, taking
TABLE 1: The parameters of distributing-flow system.

\begin{tabular}{lcc}
\hline Parameters & Value & Symbol/unit \\
\hline The radius of bent axle & 0.03 & $\mathrm{~m}$ \\
The ratio of crank and connecting link & 0.25 & $/$ \\
Crankshaft speed & 150 & $\mathrm{n} /(\mathrm{r} / \mathrm{min})$ \\
Water density & 998 & $\mathrm{~kg} / \mathrm{m}^{3}$ \\
Water viscosity & 0.001003 & $\mathrm{~Pa} / \mathrm{s}^{-1}$ \\
Inlet pressure & 0.1 & $\mathrm{MPa}$ \\
Outlet pressure & 2 & $\mathrm{MPa}$ \\
Saturated vapor pressure & 2339 & $\mathrm{~Pa}$ \\
Time step & 0.001 & $\mathrm{~S}$ \\
\hline
\end{tabular}

the viscidity and turbulence into consideration and gasliquid two-phase flow as the object of study, the transmission equation considering the content of gaseous mass is given as follows [21-24]:

$$
\frac{\partial}{\partial t}(\rho f)+\nabla \cdot(\rho \vec{V} f)=\nabla \cdot(\Gamma \nabla f)+R_{e}+R_{c},
$$

where $\rho$ is average density of gas-liquid mixture, $f$ is gaseous mass content, $R_{e}$ and $R_{c}$ denote velocity of bubble in generation and disappearance, respectively, $\vec{V}$ denotes average velocity of gaseous phase in two-dimensional flow, and $\Gamma$ denotes effective transmission efficiency.

Based on the bubble dynamic equation of RayleighPlesset, the bubble dynamics can be described by the variation of bubble radius under the surface tension term and the second derivative term in the equation neglected as follows:

$$
\begin{aligned}
\frac{d R_{B}}{d t} & =\sqrt{\frac{2\left|p_{v}-p\right|}{3 \rho_{l}}} \operatorname{sgn}\left(p_{v}-p\right) \\
\frac{1}{\rho} & =\frac{f}{\rho_{v}}+\frac{1-f}{\rho_{l}} \\
p_{v} & =p_{\text {sat }}+p_{\text {turb }},
\end{aligned}
$$


where $R_{B}$ is bubble radius, $p$ is pressure, $p_{v}$ is the critical pressure of gas, $p_{\text {sat }}$ is saturation pressure of gas, $p_{\text {turb }}$ is the pressure caused by turbulence, $\rho_{v}$ is gaseous density, and $\rho_{l}$ is average fluid density.

Combining transmission equation of mass with the equation of continuity, the relationship of density in mixture and volume fraction is described as follows:

$$
\frac{d \rho}{d t}=\left(\rho_{v}-\rho_{l}\right) \frac{d \alpha}{d t}
$$

where $\alpha$ is the gas volume fraction.

If the bubble numbers are $n$ in unit volume, the gas volume fraction with respect to bubble radius can be expressed as follows:

$$
\alpha=\frac{4 \pi n R_{B}^{3}}{3} .
$$

Substituting (9) into (8), the relationship of density in mixture and bubble dynamic is obtained as follows:

$$
\frac{d \rho}{d t}=\left(\rho_{v}-\rho_{l}\right) \sqrt[3]{36 \pi n \alpha^{2}} \frac{d R_{B}}{d t} .
$$

According to the above equations, the velocity of bubble in generation and disappearance can be described, respectively, as follows:

$$
\begin{aligned}
& R_{c}=\frac{3 \alpha \rho_{v} \rho_{l}}{R_{B} \rho} \sqrt{\frac{2\left(p_{v}-p\right)}{3 \rho_{l}}} \\
& R_{e}=\frac{3(1-\alpha) \rho_{v} \rho_{l}}{R_{B} \rho} \sqrt{\frac{2\left(p_{v}-p\right)}{3 \rho_{l}}} .
\end{aligned}
$$

The gas volume fraction is proportional to average velocity and average velocity can be denoted by turbulence energy. When the surface tension coefficient of bubble is introduced, the velocity of bubble in generation and disappearance also can be described, respectively, as follows:

$$
\begin{aligned}
& R_{c}=C_{c} f \frac{\rho_{v} \rho_{l}}{\sigma} \sqrt{\frac{2 K\left(p_{v}-p\right)}{3 \rho_{l}}} \\
& R_{e}=C_{e}(1-f) \frac{\rho_{v} \rho_{l}}{\sigma} \sqrt{\frac{2 K\left(p_{v}-p\right)}{3 \rho_{l}}},
\end{aligned}
$$

where $C_{c}$ and $C_{e}$ denote empirical constant, respectively, $C_{c}=$ $0.02, C_{e}=0.01$, and $\sigma$ is the surface tension coefficient of bubble.

3.3. Turbulence Model. Turbulence energy represents turbulent fluctuation and directly reflects dissipation and stability of fluid flow. If turbulence energy is larger in some areas, these areas will have more loss of kinetic energy and become more unstable [16]. The standard $k-\varepsilon$ turbulence modelbased on Reynolds Average Navier-Stokes equation is applied in our simulation and dissipation rate of turbulence energy defined as follows [22, 23]:

$$
\varepsilon=\frac{\mu}{\rho} \overline{\left(\frac{\partial \mu_{t}^{\prime}}{\partial x_{k}}\right)\left(\frac{\partial \mu_{i}^{\prime}}{\partial x_{k}}\right)},
$$

where turbulence viscosity $\mu_{t}$ is the function of fundamental unknown quantity Turbulent Kinetic Energy $k$ and dissipation rate $\varepsilon$ and is expressed as follows:

$$
\mu_{t}=\rho C_{\mu} \frac{k^{2}}{\varepsilon}
$$

where $k$ and $\varepsilon$ are fundamental unknown quantities in standard $k-\varepsilon$ model, respectively. For incompressible fluid, the corresponding transport equations are given as

$$
\begin{aligned}
\frac{\partial(\rho k)}{\partial t}+\frac{\partial\left(\rho k \mu_{i}\right)}{\partial x_{i}}= & \frac{\partial}{\partial x_{j}}\left[\left(\mu+\frac{\mu_{t}}{\sigma_{k}}\right) \frac{\partial k}{\partial x_{j}}\right]+G_{k} \\
& -\rho \varepsilon \\
\frac{\partial(\rho \varepsilon)}{\partial t}+\frac{\partial\left(\rho \varepsilon \mu_{i}\right)}{\partial x_{i}}= & \frac{\partial}{\partial x_{j}}\left[\left(\mu+\frac{\mu_{t}}{\sigma_{k}}\right) \frac{\partial \varepsilon}{\partial x_{j}}\right]+\frac{C_{1 \varepsilon}}{k} G_{k} \\
& -C_{2 \varepsilon} \rho \frac{\varepsilon^{2}}{k},
\end{aligned}
$$

where $G_{k}$ is the generation of turbulence energy occurred by average velocity gradient and is defined as

$$
G_{k}=\mu_{t}\left(\frac{\partial \mu_{i}}{\partial x_{j}}+\frac{\partial \mu_{j}}{\partial x_{i}}\right) \frac{\partial \mu_{i}}{\partial x_{j}},
$$

where $C_{1 \varepsilon}, C_{2 \varepsilon}$, and $C_{\mu}$ are empirical constants, respectively, $C_{1 \varepsilon}=1.44, C_{2 \varepsilon}=1.92, C_{\mu}=0.09, \sigma_{k}$ and $\sigma_{\varepsilon}$ are Prandtl values, respectively, corresponding to turbulence energy and dissipation rate, $\sigma_{k}=1.0$, and $\sigma_{\varepsilon}=1.3$.

\section{Results and Discussion}

4.1. Distribution of Turbulence Energy. The distribution of turbulence energy for one working cycle is shown in Figure 3. It can be shown at rotating-sleeve angles of $180^{\circ}$ and $360^{\circ}$, respectively. There are marks as evident highlight regions in specific area, and this phenomenon indicates that turbulence energy increases in the specific area. As shown in Figure 3, the angles of $180^{\circ}$ and $360^{\circ}$ are exactly when the interconversion between discharge and suction happens. Therefore, the flow field around highlight regions is seriously unstable to cause much loss of kinetic energy.

4.2. Velocity Distribution. The velocity distribution in $X$ direction for the distributing-flow system within one working cycle is shown in Figure 4. It is shown from Figure 4 that, at rotating-sleeve angle of $10^{\circ}$, velocity gradient and velocity magnitude increase, at the same time, the turbulence energy increases as shown in Figure 3. Additionally, at rotatingsleeve angles of $180^{\circ}$ and $360^{\circ}$, respectively, the flow velocity magnitude is a negative value around the damping groove, in which the turbulence energy increases. It is demonstrated that the flow condition around the damping groove is unstable.

Figure 5 shows velocity vector distribution around the damping groove when the rotating sleeve rotates from discharge to suction. At this moment, the plunger descends from 


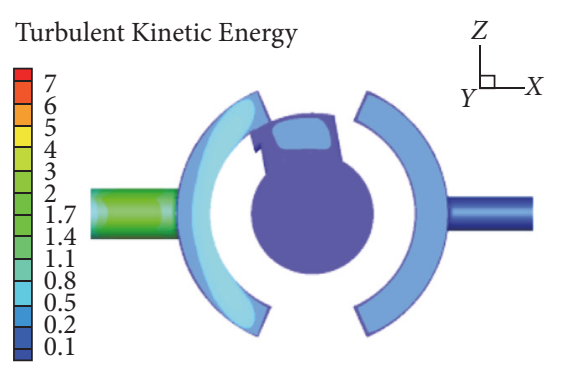

$\theta=10^{\circ}$

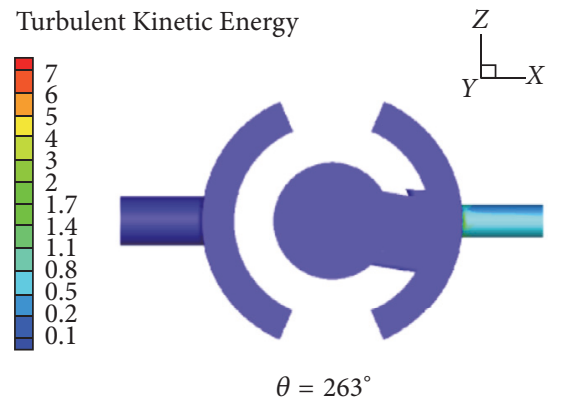

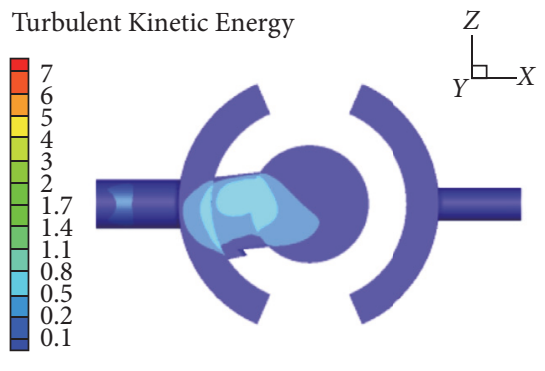

$\theta=97^{\circ}$

Turbulent Kinetic Energy

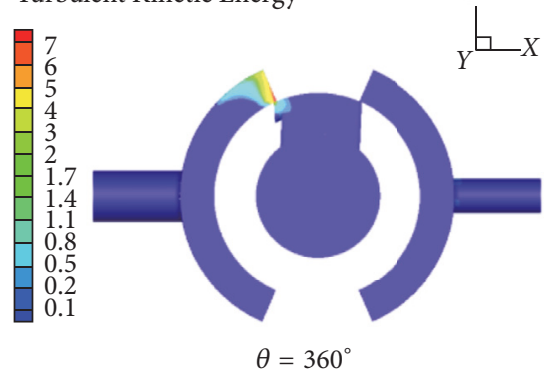

Turbulent Kinetic Energy

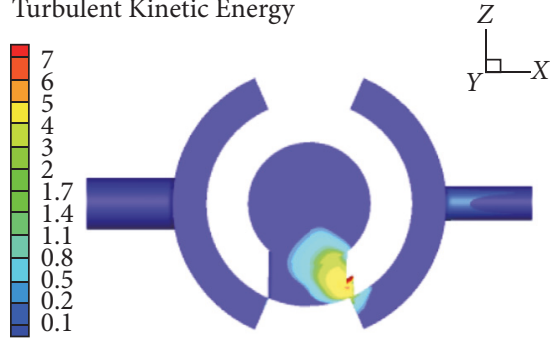

$\theta=180^{\circ}$

Turbulent Kinetic Energy

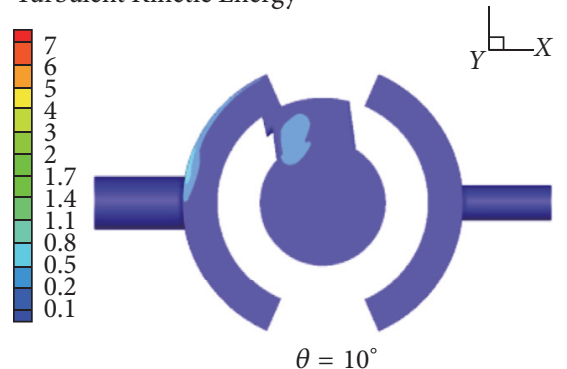

FIGURE 3: Distribution of turbulence energy for one working cycle.
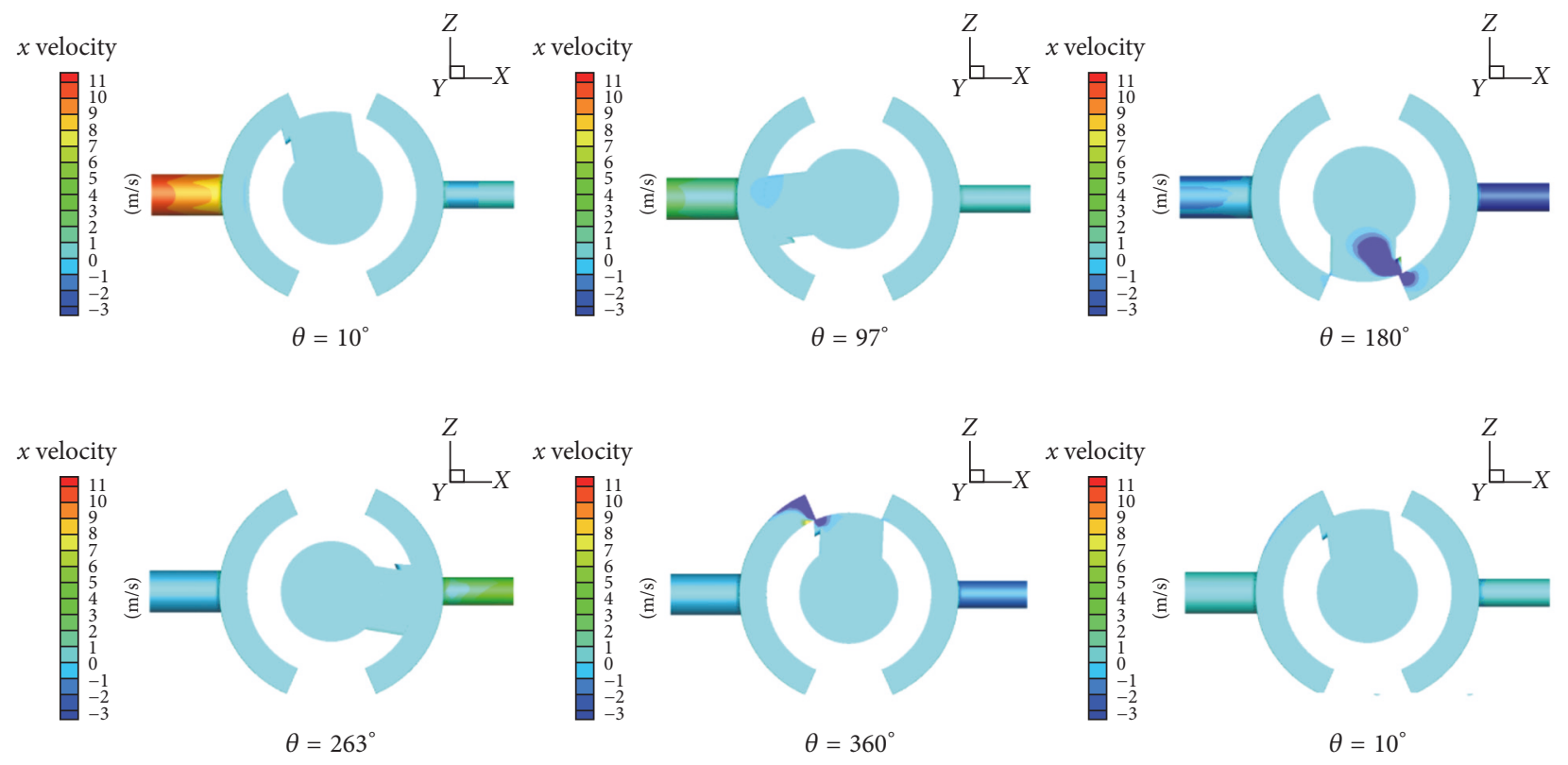

FIGURE 4: Velocity distribution in $X$ direction for one working cycle.

the TDC; the loading chamber separates from the valve port in a critical state; and there exists subtle streaming of fluid caused by inertia effect in the loading chamber as shown in Figure 5(a). Besides, the damping groove instantaneously connects with the collecting chamber and valve port as shown in Figure 5(b). The simulation results from Figure 5(b) illustrate that transitory backward flow flows from the collecting chamber into the valve port and causes dramatic turbulence around the damping groove on account of high pressure in the pump chamber as well as low pressure in the collecting chamber (differential pressure is about 1.9 MPa), in which differential pressure resulting in backward flow flowing from the collecting chamber into the pump chamber reduces volume efficiency.

Figure 6 shows local velocity vector distribution when the rotating sleeve rotates from suction to discharge. At this moment, the plunger ascends from the Bottom Dead Center (BDC); the damping groove flashily connects with 


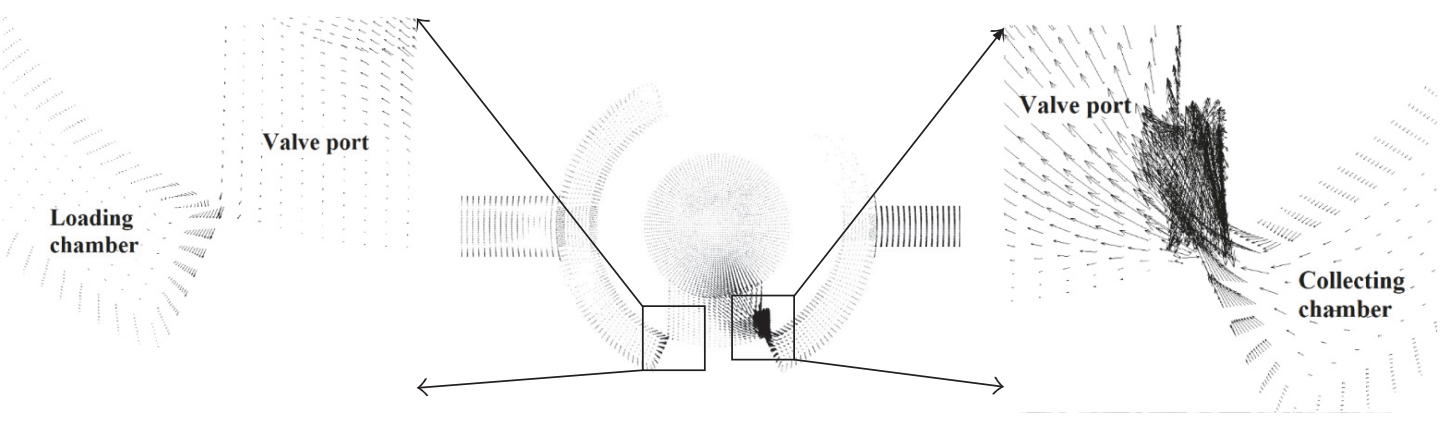

(a) Velocity vector distribution around loading chamber

(b) Velocity vector distribution around collecting chamber

FIGURE 5: Local velocity vector distribution with rotating-sleeve angle of $180^{\circ}$.

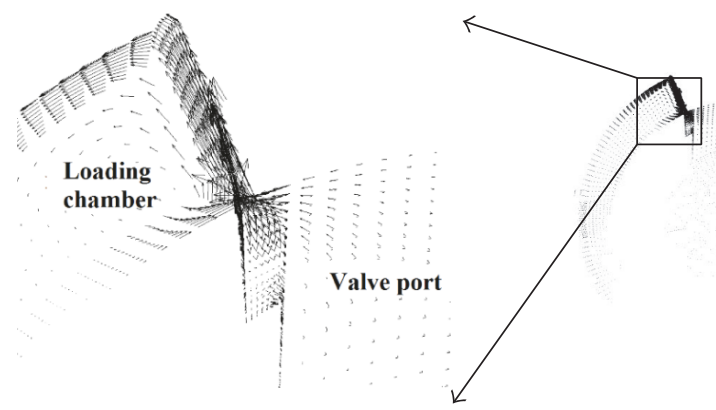

(a) Velocity vector distribution around loading chamber

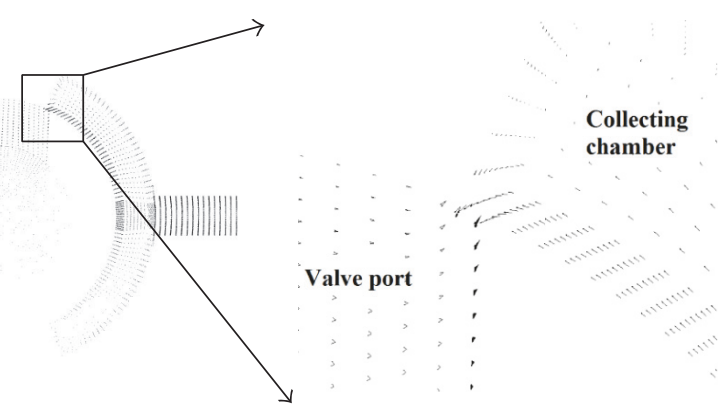

(b) Velocity vector distribution around collecting chamber

FIGURE 6: Local velocity vector distribution with rotating-sleeve angle of $360^{\circ}$.

the loading chamber and valve port as shown in Figure 6(a). Figure 6(b) shows that the transient backward flow flows from the valve port into the loading chamber causing dramatic turbulence around the damping groove on account of high pressure in the valve port as well as low pressure in the loading chamber. At the same time, differential pressure causes backward flow to flow from the pump chamber into the loading chamber, which can seriously reduce volume efficiency; the loading chamber is separating from the valve port in a critical state; and there exists subtle streaming that is caused by inertia effect in the loading chamber.

\subsection{Analyses on Working Pulsation}

4.3.1. Flow Pulsation of Different Valve Port Structures. Figure 7 demonstrates the mesh of distributing-flow system by dealing with structured mesh generation. The sliding grid option provides the grid interface for the sliding surfaces between the static inlet/outlet part of grid and the outside part of valve port, the inside part of valve port, and the static part of pump chamber. The piston movement is modeled by a dynamic grid with a moving and deforming mesh for the pump chamber part. In the grid, maximum parameter in overall mesh is 1; different parts have different local mesh parameter; total numbers of grid are 397602.

Flow pulsation is the source of noise, pressure pulsation, and vibration of plunger pump. It can have adverse impacts towards working parts, especially some precise hydraulic

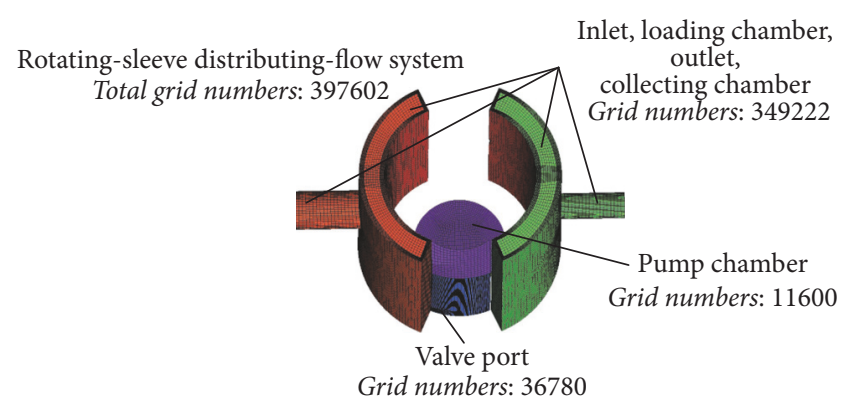

FIgURE 7: Fluid model grid of distributing-flow system.

system. Hence it should be emphasized that the characteristic of fluid is most meaningful with respect to the flow pulsation of the distributing-flow system. Figure 8 shows that outlet flow shows periodic fluctuations with maximum flow about $5 \times 10^{-4} \mathrm{~m}^{3} / \mathrm{s}$ and backward flow appears at the end of processes, that is, suction and discharge. It can be illustrated that these analyses are consistent with findings in Figures 5 and 6. Moreover, it appears as instantaneous backward flow about $2.2 \times 10^{-4} \mathrm{~m}^{3} / \mathrm{s}$ according to the left zoom in plot corresponding to the TDC in Figure 9, which includes two working cycles. Similarly, it appears as instantaneous backward flow about $2.6 \times 10^{-4} \mathrm{~m}^{3} / \mathrm{s}$ according to the right zoom in plot corresponding to the BDC in Figure 9. 


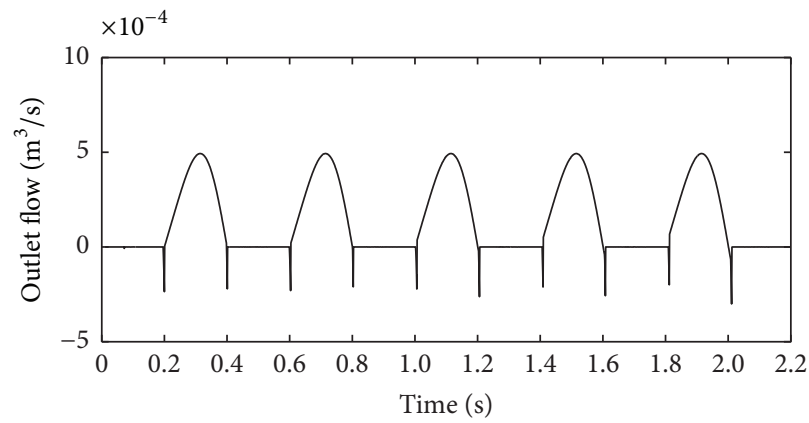

FIGURE 8: The characteristic of outlet flow pulsation.

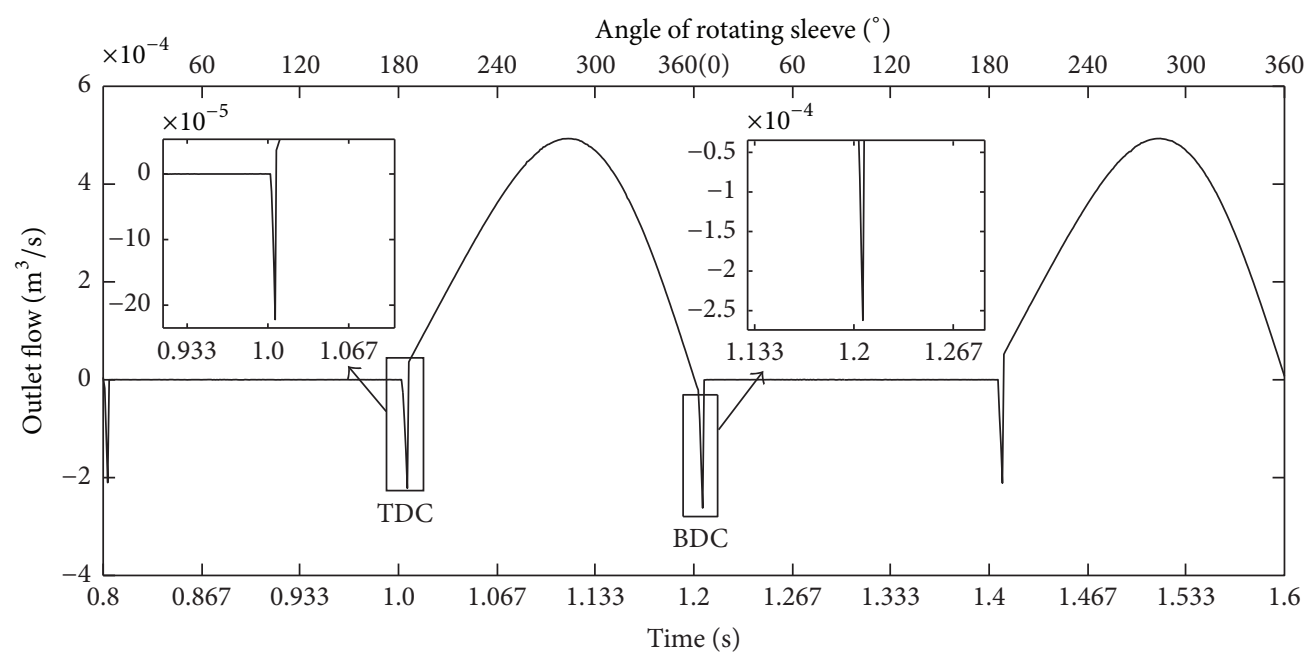

FIgURE 9: The characteristic of local outlet flow pulsation.

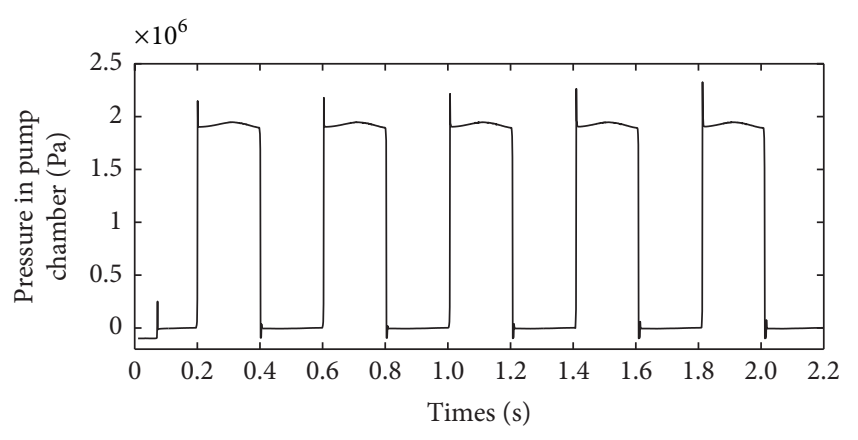

FIGURE 10: The characteristic of pressure in the pump chamber.

4.3.2. Pressure Pulsation of Different Valve Port Structures. The phenomena incorporating backward flow, local cavitation, and erosion can easily cause pressure pulsation with regard to inner flow. And once pressure pulsation happens, it can lead to intensive vibration of pump and cavitation and even resonance. Figure 10 shows periodic fluctuation and distinct sharp corner in dead center of pressure pulsations. As partial zoom in plots including two working cycles in Figure 10, left zoom in plot in Figure 11 depicts that pressure in the pump chamber quickly rises until it reaches $2.2 \times$
$10^{6} \mathrm{~Pa}$ involving over $10 \%$ more than nominal pressure when the plunger is at the TDC on account of throttling action in the damping groove. However, pressure overshoot gradually reduces to normal condition followed by increasing flow area. Because of throttling action in the damping groove, pressure in the pump chamber abruptly drops to the saturated vapor pressure about $-98.9 \mathrm{KPa}$ of fluid when the plunger is at the BDC; however, the overshoot gradually increases to the normal condition with increasing flow area.

\section{Conclusions}

(1) According to characteristic of the novel rotatingsleeve distributing-flow system, the governing equations between the plunger and rotating sleeve are established to obtain the dynamic model of distributing-sleeve system. By utilizing sliding mesh and dynamic mesh technology, the improved CFD model taking the cavitation and turbulence into consideration is employed to simulate flow field and working pulsation.

(2) Simulations of rotating-sleeve distributing-flow system have been conducted based on the governing equations and the improved CFD model. Primary 


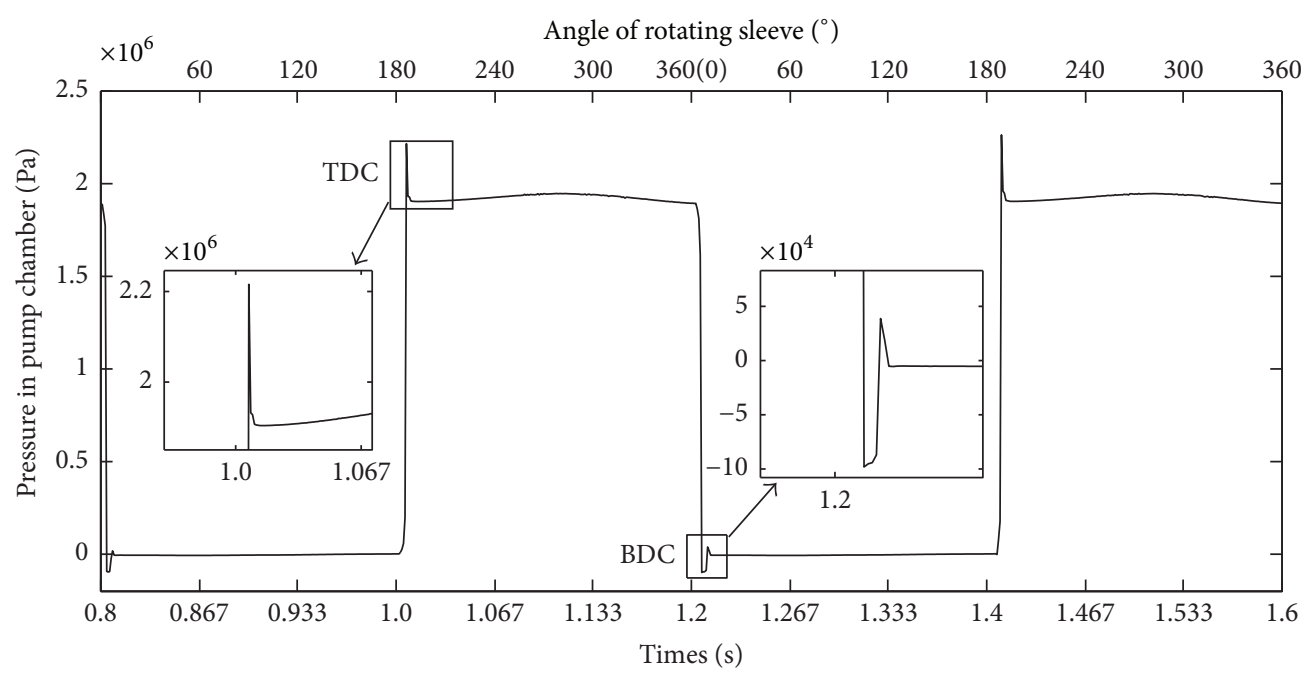

FIgURE 11: The characteristic of local pressure in the pump chamber.

performance parameters such as turbulence energy distribution, velocity distribution, and working pulsation have been investigated. In addition, the relationship between performance parameters is studied for the distributing-flow system.

(3) Periodic fluctuation and sharp corner exist in flow pulsation in addition to the backward flow issue between discharge and suction. Serious turbulence and large loss in kinetic energy around the damping groove exist. Moreover the noticeable periodic fluctuation and sharp corner appear in the pressure pulsation, and pressure in the pump chamber rapidly rises to $2.2 \mathrm{MPa}$ involving over $10 \%$ more than nominal pressure when the plunger is at the TDC. On the other hand, pressure in the pump chamber instantaneously reduces to the saturated vapor pressure $-98.9 \mathrm{KPa}$ when the plunger is at the BDC. For the rest time period, the pressure overshoot gradually reaches stability until it closes to the normal condition with increasing flow area. Fluid field and working pulsation simulation could provide foundations for structure optimization.

\section{Conflicts of Interest}

The authors declare that there are no conflicts of interest regarding the publication of this paper.

\section{Acknowledgments}

The research work was supported by National Natural Science Foundation of China (Grant no. 51575286) and Shandong Province Science Foundation of China (no. 2014ZRB01503).

\section{References}

[1] B. Zhang, J. Ma, H. Hong, H. Yang, and Y. Fang, "Analysis of the flow dynamics characteristics of an axial piston pump based on the computational fluid dynamics method," Engineering Applications of Computational Fluid Mechanics, vol. 11, no. 1, pp. 86-95, 2017.

[2] F. Yin, S. Nie, W. Hou, and S. Xiao, "Effect analysis of silencing grooves on pressure and vibration characteristics of seawater axial piston pump," Proceedings of the Institution of Mechanical Engineers, Part C: Journal of Mechanical Engineering Science, vol. 231, no. 8, pp. 1390-1409, 2017.

[3] C. Olendraru, A. Sellier, M. Rossi, and P. Huerre, "Inviscid instability of the Batchelor vortex: absolute-convective transition and spatial branches," Physics of Fluids, vol. 11, no. 7, pp. 1805-1820, 1999.

[4] I. Delbende, J.-M. Chomaz, and P. Huerre, "Absolute/convective instabilities in the Batchelor vortex: a numerical study of the linear impulse response," Journal of Fluid Mechanics, vol. 355, pp. 229-254, 1998.

[5] B. Xu, K.-M. Lee, Y. Song, Q. Wang, and H. Yang, "A numerical and experimental investigation of parametric effect on flow ripple," Proceedings of the Institution of Mechanical Engineers, Part C: Journal of Mechanical Engineering Science, vol. 229, no. 16, pp. 2939-2951, 2015.

[6] P. Dhananchezhiyan and S. S. Hiremath, "Optimization of Multiple Micro Pumps to Maximize the Flow Rate and Minimize the Flow Pulsation," Procedia Technology, vol. 25, pp. 1226-1233, 2016.

[7] K. YU, Y.-G. CHENG, and X.-X. ZHANG, "Hydraulic characteristics of a siphon-shaped overflow tower in a long water conveyance system: CFD simulation and analysis," Journal of Hydrodynamics, vol. 28, no. 4, pp. 564-575, 2016.

[8] Y. Luo, Y. Chen, H. Yang, and Y. Wang, "Study on an internallycooled liquid desiccant dehumidifier with CFD model," Applied Energy, vol. 194, pp. 399-409, 2017.

[9] J. Ma, B. Xu, B. Zhang, and H. Yang, "Flow ripple of axial piston pump with computational fluid dynamic simulation using compressible hydraulic oil," Chinese Journal of Mechanical Engineering (English Edition), vol. 23, no. 1, pp. 45-52, 2010.

[10] M. A. Delele, F. Weigler, G. Franke, and J. Mellmann, "Studying the solids and fluid flow behavior in rotary drums based on a multiphase CFD model," Powder Technology, vol. 292, pp. 260271, 2016. 
[11] S. Wang, "The analysis of cavitation problems in the axial piston pump," Journal of Fluids Engineering, Transactions of the ASME, vol. 132, no. 7, pp. 0745021-0745026, 2010.

[12] L. L. Guo, J. Geng, S. Shi, and G. S. Du, "Study of the phenomenon of water hammer based on sliding mesh method," Applied Mechanics and Materials, vol. 525, pp. 236-239, 2014.

[13] P. L. Vitagliano, M. Minervino, D. Quagliarella, and P. Catalano, "A conservative sliding mesh coupling procedure for U-RANS flow simulations," Aircraft Engineering and Aerospace Technology, vol. 88, no. 1, pp. 151-158, 2016.

[14] J. Lee, C. S. Tan, B. T. Sirakov et al., "Performance Characterization of Twin-Scroll Turbine Stage for Vehicular Turbocharger Under Unsteady Pulsating Flow Environment," Journal of Engineering for Gas Turbines and Power, vol. 139, no. 7, p. 072701, 2017.

[15] J. Lee, C. S. Tan, B. T. Sirakov et al., "Performance Metric for Turbine Stage Under Unsteady Pulsating Flow Environment," Journal of Engineering for Gas Turbines and Power, vol. 139, no. 7, p. 072606, 2017.

[16] M. V. C. Alves, J. R. Barbosa Jr., and A. T. Prata, "Analytical and CFD modeling of the fluid flow in an eccentric-tube centrifugal oil pump for hermetic compressors," International Journal of Refrigeration, vol. 36, no. 7, pp. 1905-1915, 2013.

[17] M. E. Pettersson, K. G. Weddfelt, and J.-O. S. Palmberg, "Methods of reducing flow ripple from fluid power pumps - A theoretical approach," SAE Technical Papers, 1991.

[18] A. Johansson, J. O. Palmberg, and K. E. Rydberg, "Cross angle design feature for reducing noise and vibrations in hydrostatic piston pumps," in Proceedings of the Fifth International Conference on Fluid Power Transmission and Control, 2001.

[19] K. A. Harrison and K. A. Edge, "Reduction of axial piston pump pressure ripple," Proceedings of the Institution of Mechanical Engineers, Part I: Journal of Systems and Control Engineering, vol. 214, no. 1, pp. 53-64, 2005.

[20] C. Guan, Z. Jiao, and S. He, "Theoretical study of flow ripple for an aviation axial-piston pump with damping holes in the valve plate," Chinese Journal of Aeronautics, vol. 27, no. 1, pp. 169-181, 2014.

[21] F. Kong, X. Shen, W. Wang, and S. Zhou, "Performance study based on inner flow field numerical simulation of magnetic drive pumps with different rotate speeds," Chinese Journal of Mechanical Engineering, vol. 25, no. 1, pp. 137-143, 2012.

[22] M. M. Athavale, H. Y. Li, Y. U. Jiang, and A. K. Singhal, "Application of the full cavitation model to pumps and inducers," International Journal of Rotating Machinery, vol. 8, no. 1, pp. 4556, 2002.

[23] B. E. Aunder and D. B. Spai, Lectures in Mathematical Models of Turbulence, Academic Press, London, UK, 1972.

[24] J. E. Ma, Study on Flow Ripple and Valve Plate Optimization of Axial Piston Pump, Zhejiang University, Zhejiang, China, 2009. 


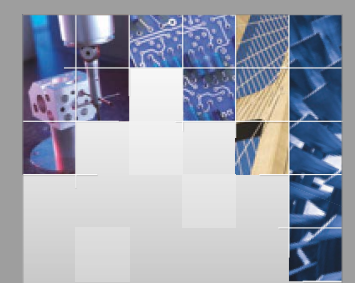

\section{Enfincering}
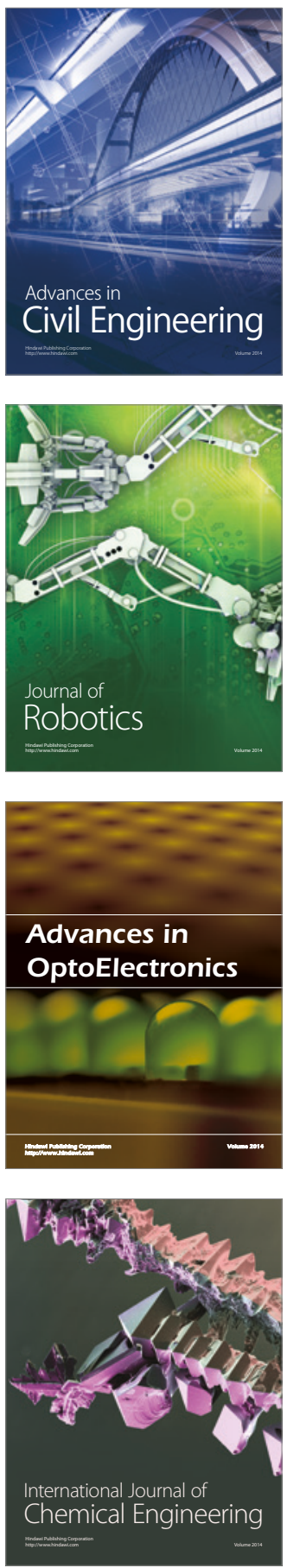

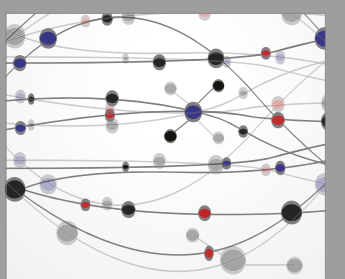

The Scientific World Journal

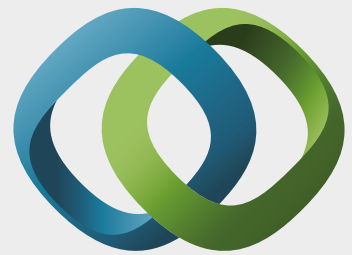

\section{Hindawi}

Submit your manuscripts at

https://www.hindawi.com
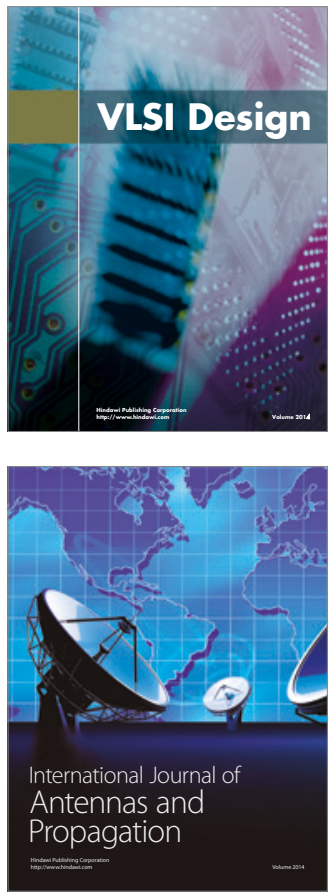

\section{Rotating}

Machinery
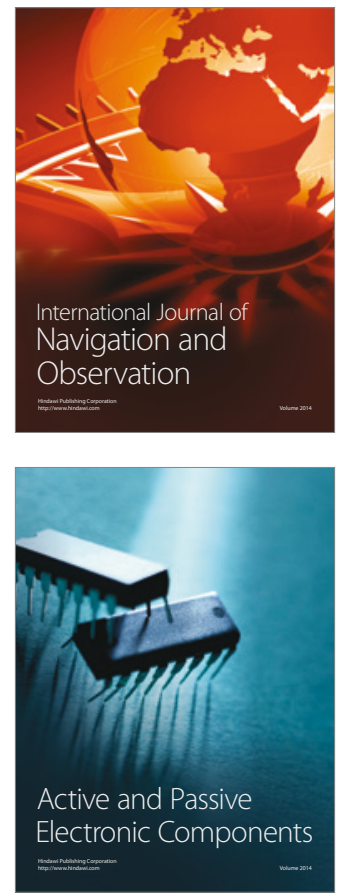
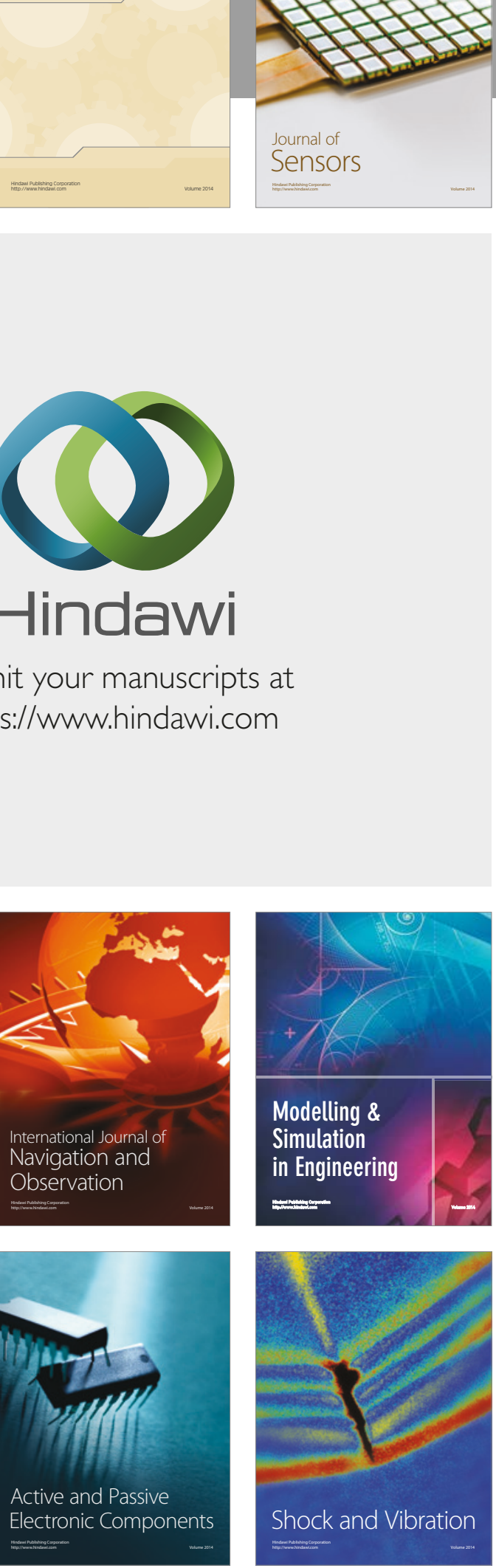
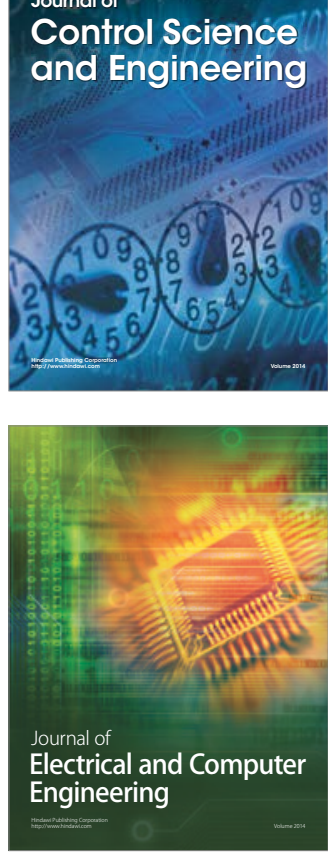

Distributed

Journal of

Control Science

and Engineering
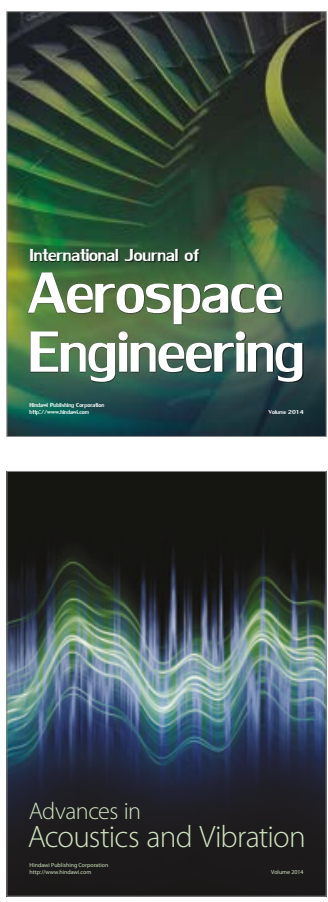

Sensor Networks 